Author Manuscript Published OnlineFirst on January 13, 2021; DOI: 10.1158/1078-0432.CCR-20-3716

Author manuscripts have been peer reviewed and accepted for publication but have not yet been edited.

Safety and Immunogenicity of LY3415244, a Bispecific Antibody Against TIM-3

and PD-L1, in Patients With Advanced Solid Tumors

Matthew D. Hellmann*, Memorial Sloan Kettering Cancer Center, New York, New York, USA, hellmanm@mskcc.org; Nicoletta Bivi, Eli Lilly and Company, Indianapolis, Indiana, USA, bivi_nicoletta@lilly.com; Boris Calderon, Eli Lilly and Company, Indianapolis, Indiana, USA, boris.calderon@lilly.com Toshio Shimizu, National Cancer Center Hospital, Chuo-Ku, Tokyo, Japan, tosshimi@ncc.go.jp; Brant R. Delafontaine, Ghent University Hospital, Gent, Belgium, email: brant.delafontaine@uzgent.be; Zhuqing Tina Liu, Eli Lilly and Company, Indianapolis, Indiana, USA, liu_zhuqing@lilly.com; Anna M. Szpurka, Eli Lilly and Company, Indianapolis, Indiana, USA, jankowska_anna_m@lilly.com; Victoria Copeland, Eli Lilly and Company, Indianapolis, Indiana, USA, Copeland_Victoria@lilly.com; F. Stephen Hodi, Dana Farber Cancer Institute, Boston, Massachusetts, USA, Stephen_Hodi@dfci.harvard.edu; Sylvie Rottey, Ghent University Hospital, Gent, Belgium, sylvie.rottey@ugent.be; Philippe Aftimos, Clinical Trials Conduct Unit, Institut Jules Bordet - Université Libre de Bruxelles, Brussels, Belgium, philippe.aftimos@bordet.be; Yongzhe Piao, Eli Lilly Japan K. K., Kobe, Japan, PIAO_YONGZHE@LILLY.COM; Leena Gandhi, Eli Lilly and Company, Indianapolis, Indiana, USA, gandhi_leena@lilly.com; Violeta Regnier Galvao, Eli Lilly and Company, Indianapolis, Indiana, USA, vgalvao@lilly.com; Ching Ching Leow, Eli Lilly and Company, Indianapolis, Indiana, USA, leow_ching_ching@lilly.com; Toshihiko Doi, National Cancer Center Hospital East, Chiba, Japan, doi.toshi@gmail.com

${ }^{*}$ Co-first authors as both authors have contributed equally to this manuscript.

Running Title: Safety and immunogenicity of LY3415244

\title{
CORRESPONDING AUTHOR
}

Matthew D. Hellmann

Memorial Sloan Kettering Cancer Center

1275 York Avenue

New York, New York 10065

Email: hellmanm@mskcc.org

Phone: $646-888-4863$

\section{Conflict of interest statement:}

$\mathrm{MDH}$ receives research support from Bristol-Myers Squibb; has been a compensated consultant for Merck, Bristol-Myers Squibb, AstraZeneca, Genentech/Roche, Nektar, Syndax, Mirati, Shattuck Labs, Immunai, Blueprint Medicines, Achilles, and Arcus; received travel support/honoraria from AstraZeneca, Eli Lilly, and Bristol-Myers Squibb; 
has options from Shattuck Labs, Immunai, and Arcus; has a patent filed by his institution related to the use of tumor mutation burden to predict response to immunotherapy (PCT/US2015/062208), which has received licensing fees from PGDx. NB, ZTL, AMS, and CCL report they are employees of Eli Lilly and Company. NB, ZTL, and LG report they are stock owners of Eli Lilly and Company. TS reports grants from Novartis, Eli Lilly, Daiichi-Sankyo, Bristol-Myers Squibb, Eisai, AbbVie, AstraZeneca, Takeda Oncology, Incyte, Chordia Therapeutics, 3D-Medicine, Symbio Pharmaceuticals, PharmaMar, Five Prime, Astellas Pharma, and Pfizer; lecture fees from Taiho Pharmaceuticals, Boehringer Ingerhim, Chugai Pharmaceuticals, and ONO Pharmaceuticals; and advisory fees from Takeda Pharmaceuticals and AbbVie. BRD reports the drug research unit at UZ Ghent received payments from Lilly for the conduct of this sponsored clinical trial. FSH reports grants and royalties to institution from BMS and Novartis; consulting fees paid from BMS, Merck, EMD Serono, Novartis, Aduro, Sanofi, Kairos, Psioxus Therapeutics, Pieris Pharmaceutical, Corner Therapeutics, Eisai, Idera, Takeda, and Genentech/Roche; advisory board fees from Surface, Compass Therapeutics, Apricity, Pionyr, 7 Hills Pharma, Torque, Rheos, Bicara, Zumutor, Checkpoint Therapeutics, and Bioentre; and equity from Apricity, Pionyr, Torque, and Bicara; patent issued and royalties paid for Methods for Treating MICARelated Disorders (\#20100111973); patents issued for Tumor antigens and uses thereof (\#7250291), therapeutic peptides (\#9402905), Vaccine compositions and methods for restoring NKG2D pathway function against cancers (\#10279021), Antibodies that bind to MHC class I polypeptide-related sequence A (\#10106611); patents pending for Angiopoiten-2 Biomarkers Predictive of Anti-immune checkpoint response (\#20170248603), Compositions and Methods for Identification, Assessment, Prevention, and Treatment of Melanoma using PD-L1 Isoforms (\#20160340407), therapeutic peptides (\#20160046716), therapeutic peptides (\#20140004112), therapeutic peptides (\#20170022275), therapeutic peptides (\#20170008962), methods of using pembrolizumab and trebananib, and únti-galectin antibody biomarkers predictive of anti-immune checkpoint and anti-angiogenesis responses publication number 20170343552. PA reports personal fees from Servier, Radius, Roche, Novartis, Macrogenics, Amcure, G1 Therapeutics, Boehringer Ingelheim, Amgen, Synthon, and MSD; and travel grants from Pfizer, Roche, Amgen, and MSD. YP reports employment with Eli Lilly Japan. TD reports grants and personal fees from Lilly, MSD, Daiichi Sankyo, Sumitomo Dainippon, Taiho, Novartis, Boehringer Ingelheim, BMS, and Abbvie; grants from Merck Serono, Janssen, Pfizer, Quintiles, and Eisai; personal fees from Oncolys BioPharma, Astellas Pharma, Ono Pharmaceutical, Rakuten Medical, Bayer, Takeda, and Amgen. BC, VC, SR, and VRG have nothing to disclose. 


\section{TRANSLATIONAL RELEVANCE}

Bispecific antibodies are a rapidly emerging therapeutic paradigm, but their structurally complex formats are often accompanied by risk of immunogenicity, including antidrug antibodies (ADAs), and downstream effects on safety and efficacy. To date, our understanding of immunogenic molecules in clinical trials is limited, as they are often discontinued from development and not reported in the public domain. Here, we report the Phase 1 clinical experience of the bispecific antibody LY3415244, where significant immunogenicity led to hypersensitivity reactions and early trial termination. ADAs were directed against both arms of LY3415244 with significantly higher titers when compared to monotherapy of the 2 parental antibodies (anti-TIM-3 and anti-PD-L1 monoclonal antibodies). Notably, higher preexisting antibodies against the bispecific compared to their respective parental monoclonal antibodies was observed in healthy donors. Overall, these findings highlight the need for early and comprehensive immunogenicity risk assessment, which includes determination of preexisting antibodies, especially when developing novel bispecific antibody structures. 


\section{ABSTRACT}

Purpose: Investigate the safety and efficacy of LY3415244, a TIM-3/PD-L1 bispecific antibody that blocks TIM-3 and PD-L1 in patients with advanced solid tumors.

Experimental Design: A Phase 1, multicenter, open-label study was conducted in patients with advanced solid tumors. Patients were dosed every 2 weeks intravenously with flat doses of LY3415244 escalating from $3 \mathrm{mg}$ to $70 \mathrm{mg}$. The primary endpoints were safety, tolerability, and identification of the recommended Phase 2 dose.

Results: Between November 2018 and October 2019, 12 patients were enrolled into 4 cohorts and received at least 1 dose of LY3415244. Two patients (16.7\%) developed clinically significant anaphylactic infusion-related reactions and all patients developed treatment emergent anti-drug antibodies (TE-ADAs). ADA titers were sometimes very high and negatively impacted soluble TIM-3 target engagement in most patients. ADA epitope specificity was against both TIM-3 and PD-L1 arms of the bispecific antibody; most TE-ADAs initially targeted the TIM-3 arm after the first dose. Pre-existing ADAs against LY3415244 were also detected in normal (unexposed) human serum samples. One patient with PD-1 refractory NSCLC had a near partial response (-29.6\%).

Conclusions: This TIM-3 and PD-L1 bispecific format was associated with unexpected immunogenicity targeting both arms of the bispecific antibody, resulting in early study termination. Epitope specificity analysis revealed an initial response towards the TIM-3 arm and presence of pre-existing ADAs to the bispecific molecule in the general population. This experience emphasizes the importance of thorough analyses for pre-existing ADAs as part of immunogenicity risk assessment of novel antibodies. 


\section{INTRODUCTION}

Programmed death (ligand) 1 (PD-[L]1) immune checkpoint inhibitors (i.e. anti-PD-1 or anti-PD-L1) have demonstrated clinical benefit in a subset of patients across a variety of tumor types, with manageable safety $(1-8)$. However, primary and acquired resistance are common, and new therapeutic approaches are needed (9). T-cell immunoglobulin and mucin-domain-containing molecule-3 (TIM-3) is a negative regulator of interferon gamma-secreting T-cells and cell-mediated antitumor immunity, is a cell surface marker for 'exhausted' T-cells following chronic exposure to antigen, and is co-expressed with PD-1 on exhausted T-cells in patients with cancer (10-12). Koyama et al. (2016) reported that TIM-3 expression is upregulated in patient tumors that progress following anti-PD-1 therapy, and in mouse models, the addition of TIM-3 antibodies overcame resistance to PD-1 blockade (13). Pre-clinical studies showed the blockade of both PD-1 and TIM-3 improved survival of tumor-bearing mice compared to blocking PD-1 only (14). The dual blockade of TIM-3 and PD-1 has also been shown to restore T-cell functionality and proliferation, resulting in a more comprehensive reversal of T-cell exhaustion $(10,12,15)$.

LY3415244 is a TIM-3/PD-L1 bispecific antibody that blocks both TIM-3 signaling on immune cells and PD-L1 on tumor cells. The bispecific format was hypothesized to provide an additional mechanism of benefit by bridging together 2 cell types expressing TIM-3 (immune cell) and PD-L1 (tumor cell). The capacity of bispecific antibodies to facilitate cell-to-cell bridging has been demonstrated using a bispecific antibody targeting anti-PD-1 and anti-PD-L1 to bridge PD-1 expressed on Jurkat cells with PD-L1 
expressed on $\mathrm{CHO}$ cells (16). Herein, we report safety and immunogenicity findings of

LY3415244 monotherapy in patients with advanced solid tumors.

\section{PATIENTS AND METHODS}

\section{Patient Population and Study Design}

Study J1C-MC-JZDA was a multicenter, nonrandomized, open-label, dose-escalation

Phase 1 study of LY3415244 in patients with advanced solid tumors with planned expansion cohorts consisting of non-small cell lung cancer, urothelial cancer, mesothelioma, and melanoma. Eligible patients had histologically or cytologically confirmed advanced or metastatic solid tumors for which standard therapy did not exist or standard therapy had failed; an Eastern Cooperative Oncology Group performance status of 0 or 1 ; at least 1 measurable lesion as defined by the Response Evaluation Criteria in Solid Tumors (RECIST) v1.1 (17); and adequate hematologic, hepatic, and renal function, and no symptomatic central nervous system malignancy or metastasis. Prior immunotherapy was permitted for patients who had not experienced severe or persistent immune-related adverse events (irAEs), especially toxicities leading to discontinuation of the prior immunotherapy.

Patients were enrolled in escalating dose cohorts driven by the modified toxicity probability interval (mTPI)-2 method (18), with a minimum of 3 patients per cohort. The primary objective was to assess the safety and tolerability and identify the recommended Phase 2 dose of LY3415244 in patients with advanced solid tumors and the secondary objective was to assess the pharmacokinetics (PK) of LY3415244. 
Exploratory endpoints included assessing the immunogenicity of LY3415244, antitumor activity, and exploring the association between biomarkers and clinical outcomes.

All patients provided written informed consent, and local Institutional Review Board approvals were obtained. The study was conducted in accordance with the Declaration of Helsinki and in compliance with the International Conference on Harmonization guidelines on Good Clinical Practice. This study is registered with ClinicalTrials.gov (NCT03752177).

\section{Study Treatment}

LY3415244 was given on Days 1 and 15 of each 28-day cycle as a 60-minute intravenous infusion in all cohorts. LY3415244 was given at $3 \mathrm{mg}, 10 \mathrm{mg}, 30 \mathrm{mg}$, and $70 \mathrm{mg}$ doses in Cohort A1-A4, respectively. Dose escalation ended at Cohort A4; therefore, LY3415244 was not given at the higher planned doses. Treatment continued until patients experienced a dose-limiting toxicity (DLT), a DLT-equivalent toxicity, unacceptable toxicity, or other discontinuation criterion were met. Dose escalation took into consideration DLT and available pharmacokinetic and pharmacodynamic data from previous dose levels.

\section{Assessments}

\section{Safety}

Safety was assessed in every patient who received at least 1 dose of LY3415244.

DLTs, treatment-emergent AEs (TEAEs), DLT-equivalent toxicities, serious adverse events (SAEs), deaths, and clinical laboratory abnormalities were graded using Common Terminology Criteria for Adverse Events (CTCAE) Version 4.0. DLTs were 
defined as possibly drug-related AEs during Cycle 1 if they met 1 of the following criteria: Grade $\geq 3$ hematologic or non-hematologic toxicity, including Grade 4 immunerelated $\mathrm{AE}$, or any other significant toxicity with exceptions including but not limited to Grade 3 inflammatory reaction attributed to a local antitumor response, any grade vitiligo or alopecia, Grade 3 or 4 lymphopenia or first occurrence of Grade 3 infusionrelated reaction (IRR) if no corticosteroid prophylaxis was given and if it resolved within 6 hours with treatment.

\section{Pharmacokinetics and pharmacodynamics}

Samples were obtained for PK and pharmacodynamic (PD) analyses at multiple time points before, during, and after dosing. Planned pharmacokinetic assessments included calculations of area under time-concentration curve (AUC), minimum serum/plasma concentration $\left(\mathrm{C}_{\min }\right)$ and approximate maximum serum/plasma concentration $\left(\mathrm{C}_{\max }\right)$ of LY3415244 from concentrations of LY3415244 in plasma; however, LY3415244 was not detectable in PK analyses and therefore results are not shown. Potential PD markers included measuring markers such as soluble PD-L1 (sPD-L1) and soluble TIM-3 (sTIM3) in serum.

\section{Biomarkers}

A newly obtained pre-treatment tumor biopsy was mandatory at baseline and prior to Cycle 2 Day 1 (C2D1) infusion for biomarker analyses. Tissue immunoreactivity for PDL1 was assessed using the Dako PD-L1 immunohistochemistry (IHC) 22C3 pharmDx kit (Agilent, Santa Clara, CA). CD8 IHC was performed using a monoclonal mouse antihuman CD8 ready-to-use antibody (Dako C8-144B) on an automated staining platform. 
TIM-3 IHC was performed using a clone against the TIM-3 extracellular domain (clone D5D5R; Cell Signaling Technology ${ }^{\circledR}$, Danvers, MA). Samples were analyzed at Clinical Diagnostics Laboratory, Eli Lilly and Company, and results were evaluated by a qualified pathologist according to prespecified interpretation guidelines. The average number of positive cells counted approximately within 5 randomly selected representative high-power microscopic fields (hpf) were reported for CD8 and TIM-3. PD-L1 expression was reported using Tumor Proportional Score (TPS), which is the percentage of viable tumor cells showing partial or complete membranous staining at any intensity. The specimen was considered negative or positive for PD-L1 expression if TPS was lower than $1 \%$ or at least $1 \%$, respectively.

Immunogenicity, epitope specificity, and pre-existing anti-drug antibody reactivity

Serum samples obtained during this study were analyzed at BioAgilytix Labs (Durham, NC, USA) to screen for (Tier 1), confirm (Tier 2), and titer (Tier 3) anti-drug antibodies (ADAs) against LY3415244 according to current regulatory guidance (Supplemental Fig 1) (19). A screening ADA (Tier 1) signal above the cut point of the assay (Supplemental Methods) signifies that the sample is a "putative positive." The specificity of the screening ADA signal is assessed by adding excess unlabeled LY3415244 during the detection step; confirmation of an ADA being present is demonstrated by competitive suppression of the screening ADA signal ("confirmatory inhibition"). Similar method used for detecting TE-ADA for LY3300054, whereby anti-drug antibodies against LY3300054 are detected instead.

A patient was evaluable for assessment of treatment-emergent ADAs (TE-ADAs) if there was at least 1 non-missing test result for LY3415244 ADA for both the baseline 
period and the post-baseline period. All percentages are relative to the total number of TE-ADA evaluable patients in each treatment group.

Pre-existing ADA reactivity was assessed as previously described (20). Briefly, 56 to 60 normal human serum samples (purchased commercially from Bioreclamation IVT) were tested (Supplemental Methods) to obtain the screening ADA signal (Tier 1). Epitope specificity studies were conducted as described above and as previously described (20).

\section{Response}

All efficacy analyses were performed on the safety population. Radiographic tumor assessments were planned at baseline and every 8 weeks after C1D1 for the first year according to RECIST v1.1 (17), until disease progression or another discontinuation criteria was met. Disease control rate (DCR) was the proportion of enrolled patients who had achieved a best overall response (BOR) of confirmed complete response (CR), confirmed partial response (PR), or stable disease (SD). Duration of response, time to response, progression-free survival, and overall survival were planned and defined in the protocol, but early termination precluded these efficacy analyses as most patients were no longer on study within a year.

\section{Statistical Analysis}

The mTPI-2 method (18) was implemented in the dose escalation. This study was designed to identify a dose level with a dose-limiting target toxicity rate of $30 \%$. The mTPI-2 method considered an equivalence interval around the target toxicity rate, and it was set to be $(25 \%, 35 \%)$ for the study. 
All safety and efficacy analyses were performed in patients who received at least 1 dose of study treatment. Descriptive statistics were used to summarize findings. The dose escalation portion of the study was not designed to make an efficacy assessment; however, all available tumor response data were documented. The database lock for this analysis was November 14, 2019. Analyses were performed in SAS version 9.4 (SAS institute, Cary, NC) and Prism 9 version 9.0 (GraphPad Software). 


\section{RESULTS}

\section{Patient Characteristics}

Between November 2018 and October 2019, 12 patients were enrolled and received LY3415244 (Table 1 and Supplemental Fig 2). Three patients were treated in each of the cohorts $\mathrm{A} 1, \mathrm{~A} 2, \mathrm{~A} 3$, and $\mathrm{A} 4$, and received $3 \mathrm{mg}, 10 \mathrm{mg}, 30 \mathrm{mg}$, or $70 \mathrm{mg}$, respectively (Supplemental Table 1). Prior to enrollment, all 12 patients had received prior systemic therapy for metastatic disease.

\section{Safety and Tolerability}

All patients received more than 1 dose of LY3415244 (Supplemental Table 2), except a patient in Cohort A4 who only received a single dose due to early study termination. Six patients $(50 \%)$ discontinued study treatment because of progression, 5 patients discontinued due to early study termination due to high TE-ADA titers with neutralizing potential, and 1 patient withdrew consent.

Two patients (1 each in Cohort A2 and A4) experienced Grade 1 or Grade 2 infusionrelated reaction (IRR, as characterized by transient chest discomfort, hypotension, appearance of edematous erythema) and suggestive of anaphylactic reactions. The anaphylactic reaction in the patient in Cohort A2 occurred upon infusion of C2D15 and resolved without any intervention; test results from a hypersensitivity test panel showed tryptase elevation. In the Cohort A4 patient, the anaphylactic reaction occurred upon receiving the C2D1 dose, and was treated with 2 courses of hydrocortisone and dexchlorpheniramine and then resolved. Of note, this patient previously experienced a suspected delayed hypersensitivity reaction during Cycle 1 that clinically manifested as 
a rash that appeared 10 days after the first dose and resolved with medication. Overall, as a result of high TE-ADA titers and anaphylactic reactions in 2 patients, the study was terminated early for safety reasons.

No DLTs were observed in evaluable patients, that is those who have received at least 1 dose of LY3415244. One patient in Cohort A2 experienced Grade $\geq 3$ TEAE (amylase increase) (Table 2 and Supplemental Table 3). SAEs were observed in 2 patients, both in Cohort A4 (intestinal stoma obstruction not related to study treatment and IRR considered related to study treatment). No patients died due to an AE either on study treatment or within 30 days of discontinuation from study treatment.

\section{Pharmacodynamics and Immunogenicity}

All patients who received LY3415244 developed treatment-emergent anti-drug antibodies (TE-ADAs) (Table 3). TE-ADAs were detected as early as 2 weeks postdosing (except in 1 patient in Cohort A3 who had pre-existing ADAs) and titers ranged from $1: 40$ to $1: 5,242,800$ (median 1:81,920) (Figure 1A and Table 3). The 2 patients with IRRs had TE-ADA titers of 1:20,480 (Cohort A2 event) and 1:655,360 (Cohort A4 event) from samples obtained prior to the anaphylactic event.

A dose-dependent accumulation of sTIM-3 was observed in Cycle 1 (Figure 1B and 1D, Day 1 through Day 29) as expected, indicating that LY3415244 initially bound to the intended target, TIM-3, and increased its half-life. However, beyond Cycle 2, there was a trend in diminishing sTIM-3 concentration in 9 out of 12 LY3415244-treated patients

(Figure 1B and 1D), suggesting a loss of target engagement. We hypothesized that TE-ADAs, which can recognize the target-binding portions of LY3415244, could 
interfere with or neutralize the LY3415244 interaction with sTIM-3. Indeed, in patients with loss of sTIM-3 target engagement, the decrease in STIM-3 correlated with increasing ADA titers (Spearman's rank correlation coefficient, $R_{s}=-0.5739, p=0.0274$ ), and was therefore suggestive of a neutralizing ADA effect against LY3415244 (Figure $1 \mathrm{C}$ and $1 \mathrm{E})$. However, this inverse relationship between increasing ADAs and decreasing sTIM-3 target engagement was not universally observed in all patients (patients 8, 11, and 12 as exceptions, Figure 1D and 1E).

Serum concentrations of SPD-L1 and LY3415244 were below the limit of quantification, limiting the capacity to examine the dynamics of these features or the correlation with ADA.

\section{ADA Epitope Specificity and Pre-existing ADA Reactivity}

Next, we conducted epitope specificity studies to determine whether the TE-ADAs were directed against the entirety of the LY3415244 versus an immune-dominant epitope in 1 arm. Patient samples were analyzed in the ADA assay in the presence of the parent monoclonal antibodies (ABC110 for PD-L1 and C22 for TIM-3) that contained the same complementarity-determining regions (CDRs) as in LY3415244. In every cohort, on average, patients developed TE-ADAs against both arms of the bispecific antibody (Supplemental Table 4). Investigation of the temporal dynamics of arm-specific ADAs suggested the anti-TIM-3 ADA response appeared earliest, present by Day 14, whereas the anti-PD-L1 immunogenicity manifested later at Day 29. By Day 56, the specificity appeared to be equally distributed between the 2 arms (Figure 2A). 
It has been shown that the incidence of clinical TE-ADA in dosed subjects correlates with detection of pre-existing ADA in the healthy donors, and that the epitope specificity of pre-existing ADA can predict the epitope preference of the clinical ADA (20). Based on this, we investigated whether an early assessment of pre-existing ADA reactivity would have informed the high immunogenicity risk of LY3415244. Sixty normal human serum samples were obtained and tested for pre-existing ADA reactivity against LY3415244. ADA assay signals (Tier 1) increased across the normal human serum samples to indicate presence of pre-existing ADA reactivity to anti-TIM-3/PD-L1 in approximately 9 out of 60 samples (Figure 2B), but precise quantification of the prevalence of pre-existing ADA reactivity was not possible due to the uncertain upper limit of normal. The ADA signal was inhibited down to the assay's background levels across all 60 samples in the presence of LY3415244 (confirmatory inhibition), indicating the presence of ADAs specific to LY3415244 (data not shown). On the other hand, preexisting ADA reactivity directed towards the 2 parental antibodies, anti-PD-L1 (LY3300054) and anti-TIM-3 (LY3321367), was comparatively very low and closer to background levels detected by the assay (Figures 2C and 2D, respectively). Similar to what was observed with the ADAs in the clinical samples from patients treated with LY3415244, pre-existing ADA immunogenicity towards LY3415244 in normal human serum samples mainly targeted the TIM-3 CDRs. In epitope specificity studies, the TIM-3 antibody variant (C22) used for the assembly of LY3415244 bispecific (TIM-3/PD-L1) inhibited the pre-existing ADAs in normal human serum samples similarly to LY3415244, whereas the PD-L1 antibody elicited a minimal inhibition, which was statistically lower compared to both LY3415244 and C22 (t-test $\mathrm{p}=0.0008$ and 
0.0044 , respectively, Figure $2 \mathrm{E})$. Together, these data indicate the specificity of preexisting ADAs in healthy donors and observed clinical ADAs was similar (compare Figure $2 \mathrm{E}$ to Figure $2 \mathrm{~A}$ ). Furthermore, and consistent with previously published reports (20), we observed that the ADA signal at baseline for each patient was fairly low and comparable to the majority of the lowest signal seen in healthy donors (Figure 2F). Only patient 8 had a higher ADA signal (290 ECLU) that resulted in a titer of 1:80 (Figure 1A).

\section{Antitumor Activity}

At study discontinuation, no patients had a complete or partial response. One patient in Cohort A1 with non-small cell lung cancer (NSCLC) who had 1 prior line of anti-PD-1 therapy and was resistant to PD-1 blockade achieved a near PR, with tumor regression of $-29.6 \%$ for 3 consecutive scans and was sustained at study discontinuation. Three patients had a BOR of SD, 8 patients had progressive disease, and 1 patient was nonevaluable. DCR was $33.3 \%$ for each of the cohorts $A 1, A 2$ and $A 3$.

\section{Biomarkers}

Tumor tissue samples were collected at baseline and on-treatment from all enrolled patients and analyzed for PD-L1, TIM-3, and CD8 expression (Supplemental Table 5). One patient with near PR had very high PD-L1 expression (TPS 90\%) at baseline, detected in archival tissue sample collected prior to any anti-PD1 therapy. PD-L1 expression was negative in 10 of 12 remaining patients. Average TIM-3 counts ranged from 0 to 65 TIM-3 positive cells per $h p f(n=11)$. 
Author Manuscript Published OnlineFirst on January 13, 2021; DOI: 10.1158/1078-0432.CCR-20-3716

Author manuscripts have been peer reviewed and accepted for publication but have not yet been edited.

The change in CD8 expression pre- versus on-treatment was assessed as well. An increase in intra-tumoral CD8 positive T-cell count was detected post-treatment in 6 of 8 evaluable patients (Supplemental Table 5 and Supplemental Figure 3). 


\section{DISCUSSION}

LY3415244 is a TIM-3/PD-L1 bispecific antibody that can target and inhibit both TIM-3 and PD-L1, which was hypothesized to overcome primary and acquired anti-PD-(L)1 resistance by a novel mechanism bridging TIM-3- and PD-L1-expressing cells. However, high ADA titers, the loss of STIM-3 target engagement suggestive of neutralizing ADA, and 2 cases of anaphylaxis, impaired the benefit-risk profile of this molecule and the study was terminated early.

ADA titers were observed in all patients who received LY3415244, with no evident dose dependency. High titers could be detected as early as C1D15 and continued to accumulate with each dose of LY3415244. The reduction seen in sTIM-3 target engagement along with increment in ADA titers is suggestive of a neutralizing ADA effect. Two patients with high ADA titers exhibited a hypersensitivity reaction consistent with anaphylaxis, although not all patients with high ADA titers manifested clinically. Interestingly, 1 patient with PD-1 refractory NSCLC (despite high PD-L1 expression) had durable tumor reduction with LY3415244 despite being in the lowest dose cohort and progressive reduction of STIM-3 target (suggestive of neutralizing ADA) with each dose.

In order to better understand whether the TIM-3 arm or the PD-L1 arm was eliciting the high ADA titers, we examined the ADA epitope specificity. We found that epitopes from both arms were immunogenic, although with distinct temporal dynamics; the response to TIM-3 epitopes occurred earlier than the response to PD-L1 epitopes, suggesting the TIM-3 arm of LY3415244 may have been the earliest and main driver of clinical immunogenicity. 
Various tools are currently available to investigate the immunogenicity of novel biotherapeutics, including assessment of pre-existing reactivity described here. Assessing the levels of pre-existing antibodies in healthy donors provides a valuable measure of the incidence of pre-existing biotherapeutic-specific B-cells and antibodies in the general population. In a patient that has not yet been dosed with a novel biotherapeutic, the presence of pre-existing antibodies indicates a potential liability of the molecule and can predict a higher incidence of immunogenicity compared to what would be seen in the absence of any pre-existing ADA.

The magnitude of preexisting ADA reactivity against a biotherapeutic assessed in treatment-naïve individuals and measured by the $90^{\text {th }}$ percentile of inhibited signal (Tier 2), correlates with the incidence of ADA in patients who have been administered the biotherapeutic (20). In our analysis, we observed pre-existing ADA immunogenicity against the bispecific molecule LY3415244 mainly directed towards the TIM-3 arm, similar to what was observed from analysis of the ADAs in the clinic. This finding was unexpected, since both parental antibodies were characterized by low pre-existing ADA reactivity. In addition, and consistent with the notion of a correlation between preexisting ADA reactivity and clinical immunogenicity, neither LY3400054 (anti-PD-L1) nor LY3321367 (anti-TIM-3) elicited a strong ADA response when administered to patients as monotherapy (Table 3). A potential explanation for the heightened immunogenicity of LY3415244 compared to its parental antibodies may be amino acid changes introduced in the heavy and light chains of CDR2 and CDR3 of the original parent antibody LY3321367 in order to generate an antibody (C22) that would maintain optimal affinity to its target, while pairing with LY3400054 to form LY3415244. We hypothesize that the 
added mutations resulted in novel B-cell epitopes in C22 not present in LY3321367, and likely contributed to the enhanced immune response to LY3415244.

However, it is important to note that, at the patient level, the presence of ADA prior to dosing is not always a direct predictor of clinical immunogenicity. Additional mechanisms of immunogenicity may be at play, including pre-existing biotherapeuticspecific T-cells. The combination of risk factors can amplify or downplay the contribution of pre-existing ADA on the unique immune response that each patient can mount against a biotherapeutic. In this study, immunogenicity was widespread across dosed patients, and not limited to the individuals with pre-existing antibodies, suggesting that the presence of pre-existing ADA on its own is not sufficient to put a patient at risk of developing treatment-induced ADA. Incorporating assessment of pre-existing antibodies can therefore improve our understanding the immunogenicity risk profile of a biotherapeutic prior to it being administered in a clinical trial, but does not address all potential issues. Future studies to identify the T-cell epitopes presented by dendritic cells upon exposure to LY3415244, and the comparison to the parent monoclonal antibodies (anti-TIM-3 mAb and anti-PD-L1 mAb), may provide additional insights into the immunogenicity response.

Nevertheless, our data suggest that determining pre-existing ADA immunogenicity to novel cancer molecules in the general population may be important for screening potential biotherapeutics prior to entering the clinical setting, in order to identify potentially immunogenic domains likely to be targeted by TE-ADA and help predict the clinical immunogenicity risk. This step may be particularly important for molecules with innovative architectures and structures, such as bispecific antibodies or fusion proteins, 
in which the risk of immunogenicity tends to be higher (20). In the specific case of LY3415244, an earlier assessment of pre-existing ADA reactivity would have predicted the clinical risk; most importantly, it would have provided valuable insight into the liability associated with certain mutations and potentially brought an alternative, less immunogenic bispecific antibody to the clinic. Alternatively, prophylaxis have been implemented to reduce the incidence of ADA. Immunosuppressants, such as methotrexate, have been shown to reduce the number of patients developing antibodies against infliximab and adalimumab (21). Although strategies to reduce immunogenicity against therapeutic agents by pre-depleting B cells with anti-CD20 monoclonal antibodies have been proposed, prospective results have not yet demonstrated success with this approach (22). One limitation to the strategy to deplete CD20 expressing B cells to prevent TE-ADAs may be that long-lived plasma cells that contribute to the main production of antibodies can be less affected by anti-CD20 depletion strategies $(23,24)$. In addition, anti-CD20 mAb treatment can itself trigger ADAs against anti-CD20 mAb, indicating that CD20 directed B cell depletion does not safeguard against possible immunogenicity $(25,26)$.

The immunogenicity observed in this study is likely not unique to this molecule, and strategies to predict features of immunogenicity will be important for efficient and safe clinical drug development in the future. Overall, our data suggest that while the strategy of targeting PD-L1 and TIM-3 remains attractive, evaluation of preexisting antibodies should be incorporated into early and comprehensive immunogenicity risk assessment. 


\section{ACKNOWLEDGMENTS}

We thank the study investigators and their support staff, as well as patients and their caregivers for participating in this study. Authors also thank, Aaron Matthew Gruver, M.D., Ph.D. for contributing immunohistochemistry data, Danni Yu and Duytrac Vu Nguyen for biomarker statistical support and Margaret Gardner for contributing to the immunogenicity data. Eli Lilly and Company contracted with Syneos Health for medical writing support provided by Andrea D. Humphries, PhD, and editing support provided by Dana Schamberger, MA.

This research was funded by Eli Lilly and Company. 
Author Manuscript Published OnlineFirst on January 13, 2021; DOI: 10.1158/1078-0432.CCR-20-3716

Author manuscripts have been peer reviewed and accepted for publication but have not yet been edited.

\section{References}

1. Bauml J, Seiwert TY, Pfister DG, Worden F, Liu SV, Gilbert J, et al. Pembrolizumab for Platinumand Cetuximab-Refractory Head and Neck Cancer: Results From a Single-Arm, Phase II Study. J Clin Oncol. 2017;35(14):1542-9. doi: 10.1200/JCO.2016.70.1524. PubMed PMID: 28328302; PubMed Central PMCID: PMCPMC5946724.

2. Blair HA. Atezolizumab: A Review in Previously Treated Advanced Non-Small Cell Lung Cancer. Target Oncol. 2018;13(3):399-407. doi: 10.1007/s11523-018-0570-5. PubMed PMID: 29785577.

3. Ferris RL, Blumenschein G, Jr., Fayette J, Guigay J, Colevas AD, Licitra L, et al. Nivolumab for Recurrent Squamous-Cell Carcinoma of the Head and Neck. N Engl J Med. 2016;375(19):1856-67. doi: 10.1056/NEJMoa1602252. PubMed PMID: 27718784; PubMed Central PMCID: PMCPMC5564292.

4. Kruger S, Ilmer M, Kobold S, Cadilha BL, Endres S, Ormanns S, et al. Advances in cancer immunotherapy 2019 - latest trends. J Exp Clin Cancer Res. 2019;38(1):268. doi: 10.1186/s13046-0191266-0. PubMed PMID: 31217020; PubMed Central PMCID: PMCPMC6585101.

5. Reck M, Rodriguez-Abreu D, Robinson AG, Hui R, Csoszi T, Fulop A, et al. Pembrolizumab versus Chemotherapy for PD-L1-Positive Non-Small-Cell Lung Cancer. N Engl J Med. 2016;375(19):1823-33. doi: 10.1056/NEJMoa1606774. PubMed PMID: 27718847.

6. Borghaei H, Paz-Ares L, Horn L, Spigel DR, Steins M, Ready NE, et al. Nivolumab versus Docetaxel in Advanced Nonsquamous Non-Small-Cell Lung Cancer. N Engl J Med. 2015;373(17):1627-39. doi: 10.1056/NEJMoa1507643. PubMed PMID: 26412456; PubMed Central PMCID: PMCPMC5705936.

7. Brahmer J, Reckamp KL, Baas P, Crino L, Eberhardt WE, Poddubskaya E, et al. Nivolumab versus Docetaxel in Advanced Squamous-Cell Non-Small-Cell Lung Cancer. N Engl J Med. 2015;373(2):123-35. doi: 10.1056/NEJMoa1504627. PubMed PMID: 26028407; PubMed Central PMCID: PMCPMC4681400. 8. Herbst RS, Baas P, Kim D-W, Felip E, Pérez-Gracia JL, Han J-Y, et al. Pembrolizumab versus docetaxel for previously treated, PD-L1-positive, advanced non-small-cell lung cancer (KEYNOTE-010): a randomised controlled trial. The Lancet. 2016;387(10027):1540-50. doi: 10.1016/s0140-6736(15)012817.

9. Schoenfeld AJ, Hellmann MD. Acquired Resistance to Immune Checkpoint Inhibitors. Cancer Cell. 2020;37(4):443-55. doi: 10.1016/j.ccell.2020.03.017. PubMed PMID: 32289269; PubMed Central PMCID: PMCPMC7182070.

10. Anderson AC. Tim-3: an emerging target in the cancer immunotherapy landscape. Cancer Immunol Res. 2014;2(5):393-8. doi: 10.1158/2326-6066.CIR-14-0039. PubMed PMID: 24795351.

11. Cheng L, Ruan Z. Tim-3 and Tim-4 as the potential targets for antitumor therapy. Hum Vaccin Immunother. 2015;11(10):2458-62. doi: 10.1080/21645515.2015.1056953. PubMed PMID: 26211834; PubMed Central PMCID: PMCPMC4635934.

12. Wherry EJ, Kurachi M. Molecular and cellular insights into T cell exhaustion. Nat Rev Immunol. 2015;15(8):486-99. doi: 10.1038/nri3862. PubMed PMID: 26205583; PubMed Central PMCID: PMCPMC4889009.

13. Koyama S, Akbay EA, Li YY, Herter-Sprie GS, Buczkowski KA, Richards WG, et al. Adaptive resistance to therapeutic PD-1 blockade is associated with upregulation of alternative immune checkpoints. Nat Commun. 2016;7:10501. doi: 10.1038/ncomms10501. PubMed PMID: 26883990; PubMed Central PMCID: PMCPMC4757784.

14. Du W, Yang M, Turner A, Xu C, Ferris RL, Huang J, et al. TIM-3 as a Target for Cancer Immunotherapy and Mechanisms of Action. Int J Mol Sci. 2017;18(3). doi: 10.3390/ijms18030645. PubMed PMID: 28300768; PubMed Central PMCID: PMCPMC5372657.

15. Jin HT, Anderson AC, Tan WG, West EE, Ha SJ, Araki K, et al. Cooperation of Tim-3 and PD-1 in CD8 T-cell exhaustion during chronic viral infection. Proc Natl Acad Sci U S A. 2010;107(33):14733-8. doi: 10.1073/pnas.1009731107. PubMed PMID: 20679213; PubMed Central PMCID: PMCPMC2930455. 
Author Manuscript Published OnlineFirst on January 13, 2021; DOI: 10.1158/1078-0432.CCR-20-3716

Author manuscripts have been peer reviewed and accepted for publication but have not yet been edited.

16. Kotanides H, Li Y, Malabunga M, Carpenito C, Eastman SW, et al. Bispecific targeting of PD-1 and PD-L1 enhances T-cell activation and antitumor immunity. Cancer Immunol Res. 2020 Oct; 8(10): 130010. doi: 10.1158/2326-6066.CIR-20-0304

17. Eisenhauer EA, Therasse P, Bogaerts J, Schwartz LH, Sargent D, Ford R, et al. New response evaluation criteria in solid tumours: revised RECIST guideline (version 1.1). European journal of cancer. 2009;45(2):228-47. Epub 2008/12/23. doi: 10.1016/j.ejca.2008.10.026. PubMed PMID: 19097774.

18. Guo W, Wang S-J, Yang S, Lin S, Ji Y. A Bayesian Interval Dose-Finding Design Addressing Ockham's Razor: mTPI-2: Cornell University Library; 2016 [cited 20188 May ]. Available from: https://arxiv.org/pdf/1609.08737.pdf.

19. Immunogenicity Testing of Therapeutic Protein Products - Developing and Validating Assays for Anti-Drug Antibody Detection: Guidance for Industry. Center for Drug Evaluation and Research (CDER) and Center for Biologics Evaluation and Research (CBER): U.S. Department of Health and Human Services Food and Drug Administration; 2019.

20. Bivi N, Moore T, Rodgers G, Denning H, Shockley T, Swearingen CA, et al. Investigation of preexisting reactivity to biotherapeutics can uncover potential immunogenic epitopes and predict immunogenicity risk. MAbs. 2019;11(5):861-9. doi: 10.1080/19420862.2019.1612699. PubMed PMID: 31099718; PubMed Central PMCID: PMCPMC6601536.

21. Garces S, Demengeot J, Benito-Garcia E. The immunogenicity of anti-TNF therapy in immunemediated inflammatory diseases: a systemic review of literature with a meta-analysis. Ann Rheum Dis. 2013 Dec; 72 (12):1947-55. doi: 10.1136/annrheumdis-2012-202220

22. Hassan R, Williams-Gould J, Watson T, Pai-Scherf L and Ira Pastan. Pretreatment with rituximab does not inhibit human immune response against the immunogenic protein LMB-1. Clin Cancer Research2004 Jan; 10: 16-8. doi: 10.1158/1078-0432.ccr-1160-3

23. Huang $\mathrm{H}$, Benoist $\mathrm{C}$ and Mathis $\mathrm{D}$. Rituximab specifically depletes short-lived autoreactive plasma cells in a mouse model of inflammatory arthritis. PNAS 2010 Mar 107 (10): 4658-63. doi.org/10.1073/pnas.1001074107

24. Hoffman K, Clauder A and Manz RA.Targeting B cells and plasma cells in autoimmune diseases. Front Imunol. 2018 Apr 9:835. doi: 10.3389/fimmu.2018.00835. eCollection 2018

25. Albert D, Dunham J, Khan S, Stansberry J, Kolasinski S, Tsai D, et al. Variability in the biological response to anti-CD20 B cell depletion in systemic lupus erythaematosus. Ann Rheum Dis. 2008 Dec; 67(12);1724-32. doi: 10.1136/ard.2007.083162

26. Casan JML, Wong J, Northcott MJ and Opat S. Anti-CD20 monoclonal antibodies; reviewing a revolution. Hum Vaccin Immunother. 2018 14(12); 2820-41. doi: 10.1080/21645515.2018.1508624

27. Patnaik A, Yap TA, Chung HC, de Miguel MJ, Bang YJ, Lin CC, et al. Safety and clinical activity of a new anti-PD-L1 antibody as monotherapy or combined with targeted therapy in advanced solid tumors: the PACT phase la/lb trial. Clin Cancer Res.Submitted.

28. Harding JJ, Patnaik A, Moreno V, Stein M, Jankowska AM, Velez de Mendizabal N, et al. A phase la/Ib study of an anti-TIM-3 antibody (LY3321367) monotherapy or in combination with an anti-PD-L1 antibody (LY3300054): Interim safety, efficacy, and pharmacokinetic findings in advanced cancers. Journal of Clinical Oncology. 2019;37(8_suppl):12-. doi: 10.1200/JCO.2019.37.8_suppl.12.

29. Bourdage JS, Cook CA, Farrington DL, Chain JS and Konrad RJ. An affinity capture elution (ACE) assay for detection of anti-druc antibody to monoclonal antibody therapeutics in the presence of high levels of drug. J Immunol Methods. 2007 Oct 327(1-2);10-7. doi: 10.1016/j.jim.2007.07.004. Epub 2007 Jul 27 


\section{TABLES}

Table 1. Baseline patient demographics and disease characteristics

\begin{tabular}{cc}
\hline Cohort A1-A4 \\
Total \\
$\mathrm{N}=12$
\end{tabular}

Sex, $\mathrm{n}$

Female

Male

Median age (range), years

$53.50(25-70)$

Race, $\mathrm{n}$

Caucasian

Asian

Tumor type, $n$

Breast cancer

$4^{a, a, b, c}$

Non-small cell lung cancer

$2^{b, b}$

Rectal cancer

$2^{b, d}$

Peritoneal sarcoma

$1^{\mathrm{b}}$

Desmoplastic small round cell tumor

$1^{\mathrm{d}}$

Ovarian cancer

Ductal adenocarcinoma of pancreas

Prior anticancer therapies, $\mathrm{n}$

Median number (IQR)

1 prior line

$\geq 3$ prior lines

Surgical procedure

Radiotherapy

Systemic therapy

12

$\mathrm{N}$ and $\mathrm{n}$ are numbers of patients. Abbreviations: IQR, interquartile range.

Histopathological diagnosis grade: ${ }^{a}$ poorly differentiated high grade, ${ }^{b}$ unable to determine, ${ }^{\mathrm{c}}$ undifferentiated high grade, ${ }^{\mathrm{d}}$ well differentiated low grade, ${ }^{\mathrm{e}}$ not reported. 


\section{Table 2. Treatment-emergent adverse events considered related to study treatment}

\begin{tabular}{|c|c|}
\hline Preferred Term ${ }^{\mathrm{a}}$, n (\%) & $\begin{array}{c}\text { Total } \\
\mathrm{N}=12\end{array}$ \\
\hline$\geq 1 \mathrm{TEAE}$ & $8(66.7)$ \\
\hline Constipation & $1(8.3)$ \\
\hline Dry mouth & $1(8.3)$ \\
\hline Nausea & $1(8.3)$ \\
\hline Fatigue & $1(8.3)$ \\
\hline Infusion-related reaction & $2(16.7)$ \\
\hline Increased amylase & $1(8.3)$ \\
\hline Increased eosinophil count & $1(8.3)$ \\
\hline Increased lipase ${ }^{b}$ & $1(8.3)$ \\
\hline Increased weight & $1(8.3)$ \\
\hline Decreased white blood cell count & $1(8.3)$ \\
\hline Decreased appetite & $1(8.3)$ \\
\hline Myalgia & $2(16.7)$ \\
\hline Muscular weakness & $1(8.3)$ \\
\hline Cough & $1(8.3)$ \\
\hline Dyspnea & $1(8.3)$ \\
\hline Alopecia & $1(8.3)$ \\
\hline Allergic dermatitis & $1(8.3)$ \\
\hline PPE syndrome & $1(8.3)$ \\
\hline Rash & $1(8.3)$ \\
\hline Maculo-papular rash & $1(8.3)$ \\
\hline
\end{tabular}

Abbreviations: PPE, palmar-plantar erythrodysesthesia; TEAE, treatment-emergent adverse event.

a TEAEs are listed by preferred term according to MedDRA Version 22.1.

${ }^{\mathrm{b}}$ Although no preplanned dose modifications were allowed, 1 patient in Cohort A2 required a dose delay due to an adverse event of Grade 4 lipase increase. 
Table 3. Characteristics of clinical immunogenicity in response to dosing with LY3415244, anti-TIM-3 Ab LY3321367, and anti-PD-L1 Ab LY3300054

\begin{tabular}{|c|c|c|c|}
\hline $\begin{array}{l}\text { Immunogenicity } \\
\text { Parameters }\end{array}$ & $\begin{array}{c}\text { Anti-TIM-3/PD-L1 } \\
\text { Bispecific } \\
\text { (LY3415244) }\end{array}$ & $\begin{array}{l}\text { Anti-PD-L1 } \\
\text { mAb } \\
\text { (LY3300054) }\end{array}$ & $\begin{array}{c}\text { Anti-TIM-3 mAb } \\
\text { (LY3321367) }\end{array}$ \\
\hline \multirow{4}{*}{$\begin{array}{l}\text { TE-ADA rate in the } \\
\text { clinic, (\%) }\end{array}$} & \multirow{4}{*}{12 out of $12(100 \%)$} & \multirow{2}{*}{$\begin{array}{c}\text { Monotherapy: } \\
17 \%\end{array}$} & Monotherapy ${ }^{b}$ : \\
\hline & & & \\
\hline & & \multicolumn{2}{|c|}{ Combo therapy TIM-3 mAb and PD-L1 mAb } \\
\hline & & \multicolumn{2}{|c|}{$\sim 50 \%$} \\
\hline \multirow{4}{*}{$\begin{array}{l}\text { TE-ADA Max Titer, } \\
\text { Range, (Median) }\end{array}$} & \multirow{4}{*}{$\begin{array}{l}1: 40 \text { to } 1: 5,242,880 \\
\text { (median } 1: 81,920 \text { ) }\end{array}$} & Monotherapy ${ }^{a}$ : & Monotherapy: \\
\hline & & $\begin{array}{c}1: 20 \text { to } 1: 160 \\
(1: 80)\end{array}$ & $\begin{array}{c}1: 40 \text { to } 1: 1280 \\
(1: 80)\end{array}$ \\
\hline & & \multicolumn{2}{|c|}{ Combo therapy TIM-3 mAb and PD-L1 mAb } \\
\hline & & \multicolumn{2}{|c|}{$\begin{array}{c}1: 40 \text { to } 1: 20480 \\
(1: 160)\end{array}$} \\
\hline \multirow{4}{*}{$\begin{array}{l}\text { Infusion Related } \\
\text { Reactions, (\%) }\end{array}$} & \multirow{4}{*}{$\begin{array}{c}\text { Monotherapy: } \\
2 \text { out of } 12(17 \%)\end{array}$} & \multirow{2}{*}{$\begin{array}{c}\text { Monotherapy }{ }^{a}: \\
0 \%\end{array}$} & Monotherapy ${ }^{\mathrm{b}}$ : \\
\hline & & & 1 out of $23(4 \%)$ \\
\hline & & \multicolumn{2}{|c|}{ Combo therapy TIM-3 mAb and PD-L1 mAb } \\
\hline & & \multicolumn{2}{|c|}{3 out of $18(16.7 \%)$} \\
\hline
\end{tabular}

\footnotetext{
${ }^{a}$ Reference (27)

${ }^{\mathrm{b}}$ Reference (28)

${ }^{\mathrm{C}}$ Updated data from reference (28)
}

Abbreviations: ACE, affinity capture elution; mAb, monoclonal antibody; PD-L1, programmed death ligand 1; TE-ADA, treatment-emergent antidrug antibody; TIM-3, T cell immunoglobulin and mucin-domain-containing molecule-3. 


\section{FIGURE LEGENDS}

Figure 1. Treatment-emergent anti-drug antibody titers and sTIM-3 concentrations A) ADA titers over time in all TE-ADA positive patients treated with LY3415244 (Cohorts A1 $3 \mathrm{mg}$ [red plots, patients 1-3], A2 $10 \mathrm{mg}$ [blue plots, patients 4-6], A3 $30 \mathrm{mg}$ [green plots, patients 7-9], and A4 70 mg [black plots, patients 10-12]). Patient 12 ADA titer profile (1:20 at Day 29 and 1:320 at Day 58) overlaps with patient 5 ADA titer profile. Patients with infusion-related reaction $\left({ }^{*}\right)$ and with partial response $(\ddagger)$ are labeled. B) Concentrations of sTIM-3 over time from TE-ADA positive patients with a trend in diminishing sTIM-3 target engagement concentrations over time (patients 1-7, 9, and 10). C) Correlation analysis of paired ADA titer and sTIM-3 in TE-ADA positive patients from (B). Spearman correlation coefficient $\left(R_{s}\right)$ and 2-tailed $P$ value reveals that as ADA titer increases, sTIM-3 decreases. D) Concentrations of sTIM-3 over time from TE-ADA positive patients without a trend in diminishing STIM-3 target engagement concentrations over time (patients 8, 11, and 12). E) Correlation analysis of paired ADA titer and sTIM-3 in TE-ADA positive patients from (E). Spearman correlation coefficient $\left(R_{s}\right)$ and 2-tailed $P$ value indicates no compelling correlation of ADA titer and STIM-3 concentrations. Abbreviations: ADA, anti-drug antibody; P, $P$ value; $R_{s}$, Spearman correlation coefficient; sTIM-3, soluble T-cell immunoglobulin and mucin-domaincontaining molecule-3.

Figure 2. Anti-drug antibody epitope specificity and assessment of preexisting antibodies 
A) Average confirmatory percent inhibition (Tier 2 analysis) of the ADA signal by the bispecific TIM-3/PD-L1 antibody or the monospecific anti-TIM-3 (C22) or anti-PD-L1 antibodies (ABC110) from post-baseline samples across all cohorts over time. Data are mean \pm SEM. Sixty normal human serum samples were evaluated for pre-existing ADA reactivity against B) the bispecific anti-TIM-3/PD-L1 antibody (LY3415244), C) the monospecific anti-TIM-3 antibody (LY3321367), or D) the anti-PD-L1 antibody (LY3300054). Data show the observed screening ADA signal, expressed as ECLU. E) The specificity of the epitopes targeted by the pre-existing ADA reactivity to LY3415244 was characterized through confirmatory assays in 6 normal human serum samples that showed the highest pre-existing ADA reactivity (see panel B: the last 6 samples on the right of the graph). Only the C22 variant of the anti-TIM-3 antibody (LY3321367) could inhibit the screening ADA signal against LY3415244, indicating the specificity of the preexisting ADA reactivity against the TIM-3 arm of the bispecific (LY3415244). ${ }^{*} p<0.05$ by t-test; F) Screening ADA Signal (ECLU) at Baseline in the 12 patients in this study.

Abbreviations: Ab, antibody; ECLU, electrochemiluminescent units; mAb, monoclonal antibody; PD-L1, programmed death ligand 1; TIM-3, T cell immunoglobulin and mucindomain-containing molecule-3. 
Author Manuscript Published OnlineFirst on January 13, 2021; DOI: 10.1158/1078-0432.CCR-20-3716 Author manuscripts have been peer reviewed and accepted for publication but have not yet been edited.
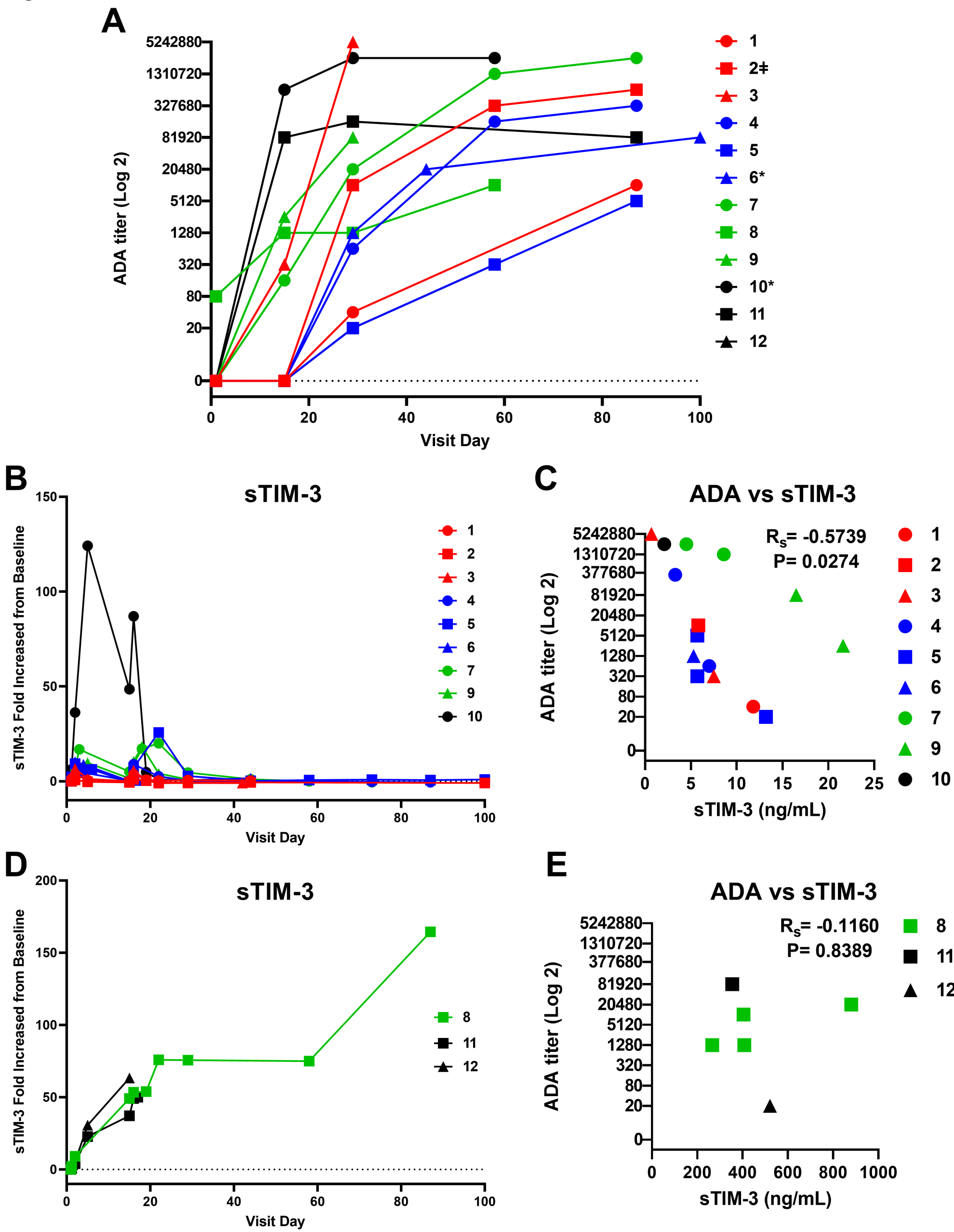

$\mathbf{E}$

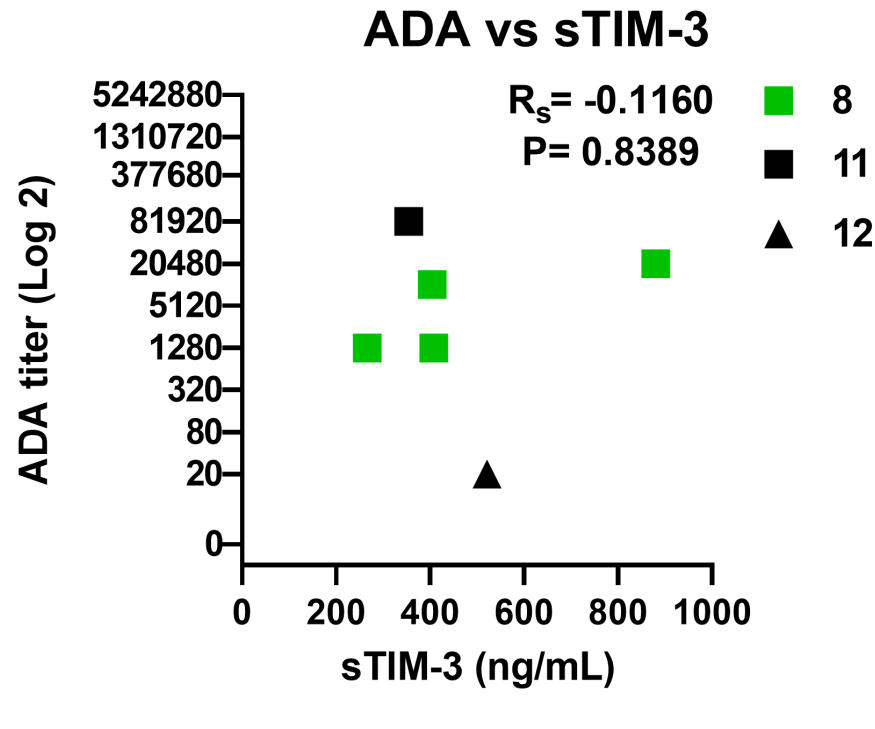

Downloaded from clincancerres.aacrjournals.org on April 6, 2021. () 2021 American Association for Cancer Research. 
Figure 2

Author manuscripts have been peer reviewed and accepted for publication but have not yet been edited.
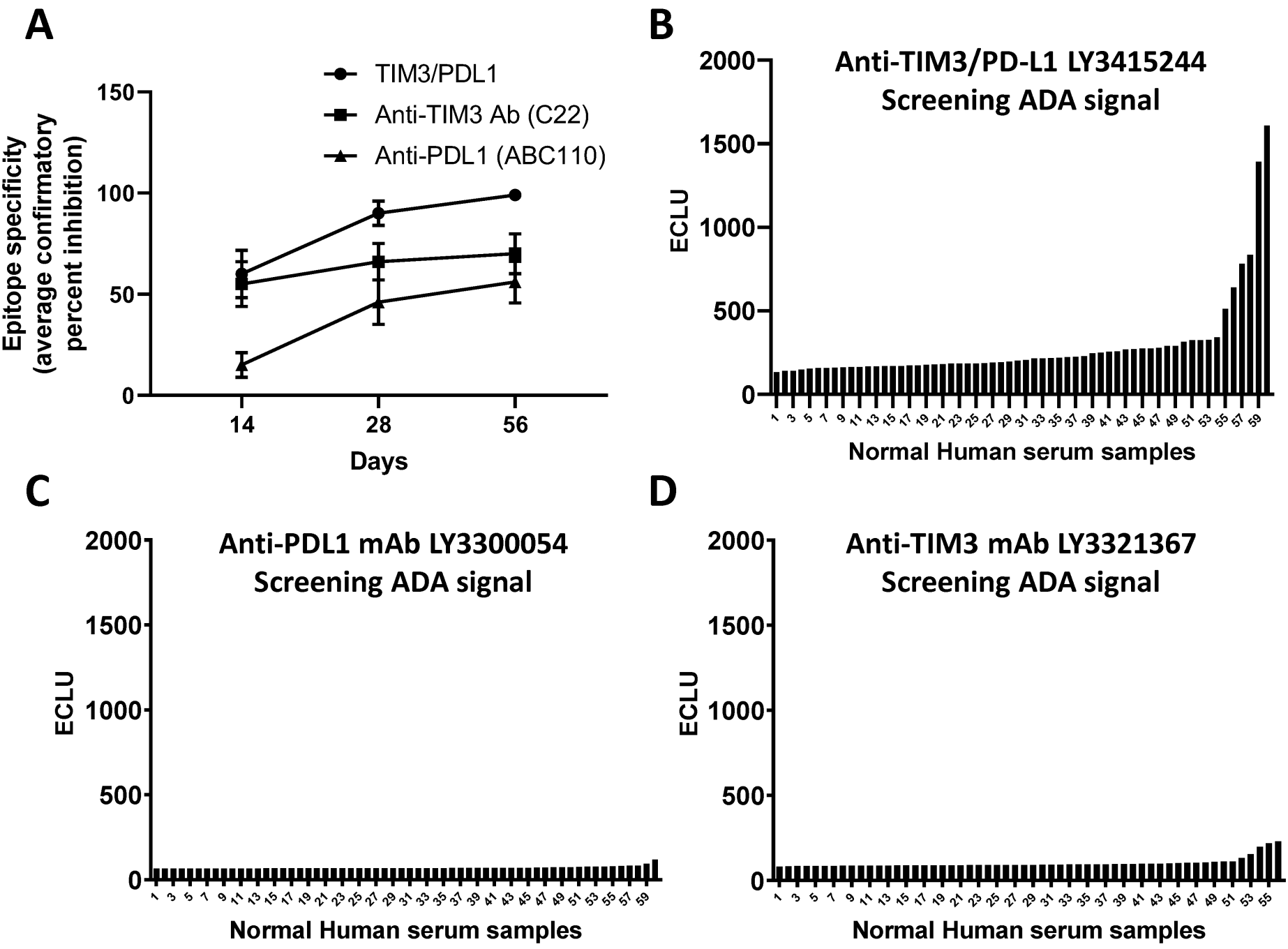

E

\begin{tabular}{cc} 
F & \\
\cline { 2 - 2 } Patient & $\begin{array}{c}\text { Screening ADA Signal } \\
\text { at Baseline (ECLU) }\end{array}$ \\
1 & 101 \\
2 & 119 \\
3 & 87 \\
4 & 71 \\
5 & 77 \\
6 & 112 \\
7 & 78 \\
8 & 290 \\
9 & 89 \\
10 & 88 \\
11 & 92 \\
12 & 73 \\
\hline
\end{tabular}

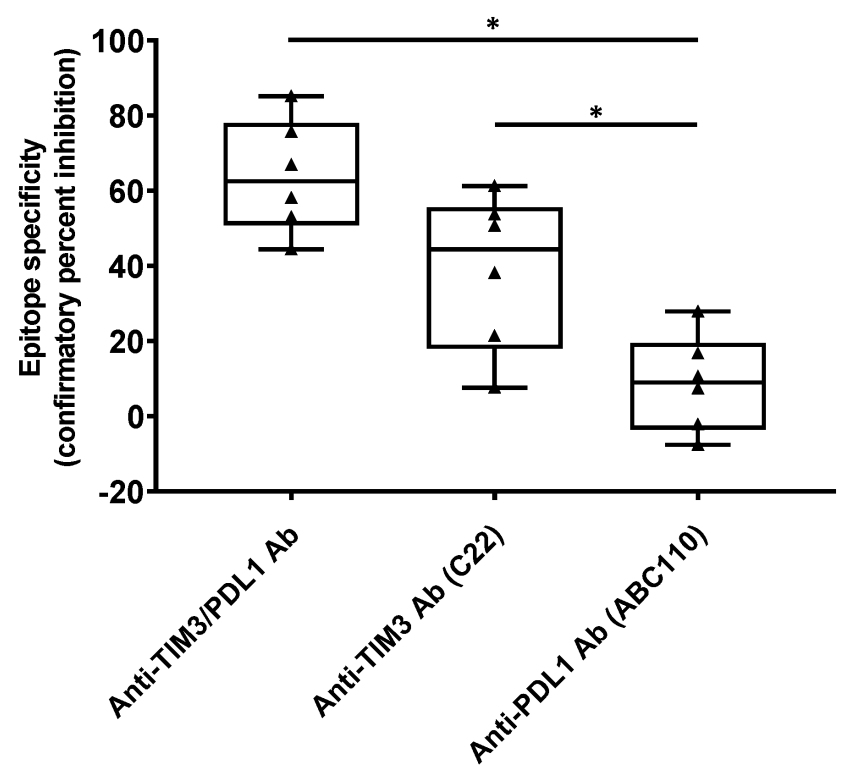




\section{Clinical Cancer Research}

\section{Safety and Immunogenicity of LY3415244, a Bispecific Antibody Against TIM-3 and PD-L1, in Patients With Advanced Solid Tumors}

Matthew D. Hellmann, Nicoletta Bivi, Boris Calderon, et al.

Clin Cancer Res Published OnlineFirst January 13, 2021.

Updated version Access the most recent version of this article at: doi:10.1158/1078-0432.CCR-20-3716

Supplementary Access the most recent supplemental material at:

Material http://clincancerres.aacrjournals.org/content/suppl/2021/01/09/1078-0432.CCR-20-3716.DC1

Author Author manuscripts have been peer reviewed and accepted for publication but have not yet been Manuscript edited.

E-mail alerts Sign up to receive free email-alerts related to this article or journal.

Reprints and To order reprints of this article or to subscribe to the journal, contact the AACR Publications Subscriptions Department at pubs@aacr.org.

Permissions To request permission to re-use all or part of this article, use this link http://clincancerres.aacrjournals.org/content/early/2021/01/12/1078-0432.CCR-20-3716. Click on "Request Permissions" which will take you to the Copyright Clearance Center's (CCC) Rightslink site. 